\title{
XI.
}

\section{L'Esquisse d'une Histoire générale et comparée des Philosophies médiévales de M. François Picavet.}

Par

\section{Albert Leclère,}

Docteur ès-lettres, Privat-Docent à l'Université de Berne.

Parmi les contemporains, M. François Picavet est l'un des historiens de la philosophie auxquels on doit le plus de reconnaissance. En 1891, il publiait un volume considérable sur ces Idéologues dont l'étude avait été dédaignée ou mal faite jusqu'à lui, même en France. On méconnaissait la relation intime du grand mouvement philosophique actuel avec les théories souvent fragmentaires, malhabiles ou bizarres, mais parfois profondes de ces penseurs si divers, parmi lesquels la foule des épigones empèchait de discerner les hardis initiateurs; et l'on oubliait l'influence décisive qu'ils exercèrent sur l'organisation de notre enseignement. Cette année, M. P. publie, non pas encore une Histoire générale et comparée des Phjlosophies médiévales, mais une Esquisse de cette histoire, dont il a déjà élaboré certaines parties en érudit que rien ne rebute, qui sait l'importance de toute idée et des circonstances de sa découverte, de son enseignement et de sa diffusion.

On possédait sans doute, sur ce sujet, des monographies plus ou moins étendues, et de réelle valeur. Mais, à part les remarquables productions d'Teberweg et surtout d'Hauréau, et l'œuvre récente de IJ. de Wulf, il n'y avait pas de travaux d'ensemble valant ces monographies. Les auteurs des meilleurs manuels d'histoire de la philosophie, ou se reproduisaient les uns les autres, ou exécutaient des variations nouvelles sur un fond d'anciens 
préjugés. A vrai dire, le courage manquait aux érudits pour aborder l'examen détaillé et l'ćtude d'ensemble d'une littérature immense, comprenant toute l'histoire politique, civile et ecclésiastique, hagiographique, thćologique et philosophique, proprement littéraire et scientifique des longs siècles au cours desquels la pensée grecque, la pensée chrétienne, la pensée juive et, à partir d'un certain moment, la pensće arabe agirent et réagirent à peu près sans interruption les unes sur les autres. Pourtant, l'on ne peut faire l'histoire de la philosophie au Moyen-Age qu'en faisant celle de toutes les philosophies qui s'y produisirent, et on nè la peut mener à bonne fin sans faire appel à toute l'histoire générale de cette époque. D'autre part, les idées et jusqu'aux institutions actuelles requièrent une telle préface. Enfin, tout autant que la spéculation antique et que la spéculation moderne, celle du Moyen-Age doit être connue pour que soit enfin possible une psychologie relativement compléte de l'homme. Comment contester la nécessité, à ce point de vue, d'une histoire sans lacunes de la pensée humaine? Celui qui considére avec impartialité la mêlée des métaphysiques, fût-il aussi éloigné que possible du scepticisme, est obligé de reconnaître que l'histoire de la philosophie, en grande partie, a pour principal intérêt d'édifier une théorie de la fantaisie dialectique de l'homme fantaisie qui tantôt prélude à la résolution méthodique de véritables problèmes, tantôt représente, en face de la science réelle, la survivance du stade de l'hypothèse pure, comme on voit encore la poésie, dans le siècle du savoir positif, coexister, ainsi qu'un monde à part, avec l'univers du physicien.

Voici les points sur lesquels l'œuvre de M. P. rompt le plus décidément avec les conceptions historiques courantes au sujet des philosophies médiévales.

Pour lui, le Moyen-Age commence réellement bien avant que l'antiquité soit close. Aux anciennes limites (ann. 355 ou 476 et 800 ou 1453), il substitue hardiment, d'une part les premiers essais de philosophie théologique que l'on peut constater au II siècle av. J. C., dans Aristobule, ou même dans le second livre des Macchabées et le livre de la Sagesse, essais résumés dans Philon, contemporain de Jésus, et d'autre part le commencement de la 
seconde moitié du XVII siècle, où la science nouvelle, la véritable science devient la principale inspiratrice de la philosophie. Ce qui lui semble caractériser au plus haut point les philosophies médiévales, c'est, en opposition avec l'esprit essentiellement naturaliste de la pensée grecque, la prépondérance des préoccupations religieuses et théologiques. Sur ce point, l'accord existait déjì entre les historiens, mais M. P. innove en ceci, qu'il fait une époque unique de tous les temps où la spéculation philosophique peut être ainsi caractérisée. Antérieure chez les Juifs à l'avénement du Christianisme, cette prépondérance s'établit au sein du monde grec à mesure que diminue l'originalité da vieil esprit hellénique; elle s'accroît rapidement dans le monde latin, en raison des dispositions religieuses et pratiques de la race; elle règne incontestablement, jusqu'au seuil des temps nouveaux, sur toute la spéculation chrétienne orientale et occidentale, sur les spéculations juives et arabes; elle s'accuse fortement encore chez une partie des penseurs de la Renaissance et des adhérents de la Réforme; elle est visible dans une partie des travaux entrepris, au XVII siècle, en opposition avec la Scolastique traditionelle; enfin, tandis que la métaphysique allemande moderne transpose dans le mode laïc, si l'on peut ainsi s'exprimer, nombre de croyances religieuses et même mystiques du Moỵen-Age, une Néo-Scolastique se prépare et se donne carrière; les plus récents philosophes professent encore, très souvent, des idées chères au Noyen-Age, ou tout au moins se posent les mêmes problèmes philosophiques sous une forme à la constitution de la quelle ont travaillé les penseurs qu'ils songent le moins à rejoindre.

Mais le souci prépondérant de la religion et de la théologie n'est pas la seule caractéristique des philosophies médiévales, que ce trait commun rapproche, en somme, plus que ne les séparent leurs divergences doctrinales ou même leurs origines confessionelles différentes. Contrairement à une opinion très répandue, jamais en Orient l'activité philosophique ne s'interrompit, et toujours elle se rattacha à la culture antique. En Occident, à part la période d'engourdissement qui va du VI au VIII siècle, des livres anciens assez nombreux sont appréciés et commentés; dès le IX siècle, leur nombre va croissant. D'une manière générale, enfin, on ne cesse guère 
de s'intéresser à la conservation et ì l'accroissement de ce que l'on connait de la science et des techniques des Anciens. Des historiens récents, entre autres M. Berthelot, ont rendu justice aux efforts du Moyen-Age dans ce domaine. Mais il ne faudrait pas, pour mieux rćagir contre l'injustice des historiens d'hier, exagérer la valeur des connaissances scientifiques médiévales, chrétiennes, juives ou arabes. Quoi qu'il en soit, il est hors de doute que l'on s'appliqua, dans les trois sortes d'écoles, à mettre la science d'accord, telle qu'on la trouvait ou la faisait, avec la philosophie et la théologie, comme on âchait d'accorder cette philosophie et cette théologie, entre lesquelles, d'ailleurs, on ne distinguait pas toujours nettement. La Scolastique déclina, mais non pour mourir, quand, une science nouvelle étant née, difficile à concilier avec la philosophie et la théologie traditionelles déjà solidarisées, on se crut fondé à la condamner en leur nom. Au lieu d'imiter Aristote, qui édifiait sa philosophie sur le savoir de son temps, on repoussa les découvertes récentes au nom de la philosophie du passé et de la théologie, en attendant que, plus tard, la science nouvelle s'étant imposée à tous, on s'efforçât de plier cette dernière aux disciplines léguées par le Moyen-Age. Tel est le rapport de la Scolastique de jadis à la Néo-Scolastique.

Certains croient avoir résumé toute la Scolastique quand ils ont signalé la part qui revient à Aristote dans les idées philosophiques du Moyen-Age. En réalité, Aristote a fourni bien des opinions aux penseurs de cette époque; mais, tout compte fait, c'est plus encore par sa forme que par sa matière, que la philosophie médiévale rappelle la pure doçtrine du Stagyrite. En effet, comment fut-il d'abord connu? Surtout par des commentateurs qui étaient plus ou moins imprégnés de Néoplatonisme. De plus, les Pères 'antérieurs à S. Augustin sont tous, directement ou indirectement, les disciples plus ou moins conscients des Platoniciens, des Stoïeiens et des Néoplatoniciens. L'Académie moyenne et la nouvelle ne furent pas sans influence sur eux. Ils sont contemporains des survivants de toutes les écoles antiques, des fidèles attardés de l'Epicurisme et des rénovateurs du Pythagorisme. L'antique philosophie est assez vivante au sein de l'Eglise pour y engendrer des: 
hérésies; et les nécessités de la polémique font que les apologistes cherchent partout des armes pour confondre leurs adversaires. Avec S. Augustin, qui doit en partie sa conversion à la lecture d'une traduction de Plotin, avec tous les mystiques et tous les docteurs des premiers siècles, parmi lesquels il faut citer au premier rang le Pseudo-Denis, le Néoplatonisme, qui avait tant d'affinités avec le Christianisme, pénètre celui-ci plus que ne fait toute autre doctrine ancienne. Cette pénétration n'était-elle point fatale, puisque, M. P. le montre avec la dernière clarté, ilyá tel passage capital de Plotin (VI. Ennéade, 9e livre), qui n'est qu'un commentaire, original sans doute, et génial, et très platonicien, mais enfin un commentaire d'un discours de S. Paul (Act. XVII, 27, 28)? Si l'on songe, enfin, que ce même Néoplatonisme avait également envahi les spéculations juives et arabes, dont la spéculation/chrétienne est si largement tributaire, on ne peut plus nier qu'il ne soit inexact de voir uniquement, dans la Scolastique, comme un prolongement, ụne survivance du Péripatétisme.

On a singulièrement exagéré, aussi, l'absence de liberté d'esprit au Moyen-Age. En effet, la forte croyance d'alors à l'harmonie nécessaire de la raison et de la foi, avait pour effet immédiat de donner, à beaucoup d'esprits, une grande confiance dans la dialectique. A tel point que, bien souvent, on ne songeait pas à distinguer les deux domaines; ou n'hésitait pas à se poser philosophiquement les nombreux problèmes dont la théologie fournissait l'idée. Et l'on voyait, dans la philosophie, le chemin qui mène à la théologie; ou bien encore on abordait celle-ci comme un ensemble de données à éclaircir, et dont l'explication pouvait être telle que la raison n'éprouvât plus, à croire, aucune difficulté. De ces deux points de rue, qui favorisaient également la libre spéculation, le premier n'était pas sans dangers, et le second était à demi hérétique. Un peu partout, de nombreux docteurs en préférèrent d'abord et longtemps encore un autre: partant de la croyance à l'inspiration des Livres Saints, il se livraientimitant en cela tous les philosophes du paganisme finissant, à l'explication allégorique, qui permettait à l'imagination intellectuelle de se déployer grandement. Quant au «Magister dixit», il ne sévit qu'assez tard dans l'évolution de la Scolastique. 
Et le champ de la recherche était bien plus vaste qu'on ne le dit d'ordinaire. Surtout, les préoccupations de la pensée médiévale n'eurent point l'uniformité dont on parle sans cesse; même, on se trompe souvent dans l'indication des principales. La connaissance toujours croissante que l'on acquérait des doctrines antiques, et la nécessité de défendre, de tous les côtés, la théologie, la christologie et la morale orthodoxes, obligèrent à traiter de presque toutes les questions qui nous occupent encore. Spécialement, le problème de la liberté, agité avec ampleur par S. Augustin, prend, ‘̀ partir de la fin du VIII siècle, une importance que n'aura certes pas, de 1090 à 1160, le problème des universaux, et qui n'a d'égale que celle du grand débat auquel donna lieu, ultérieurement, la théorie panthéistique de "l'unité de l'intellect possible» soutenue .par Averroës.

Il semble ì M. P. qu'une étude impartiale et complète des philosophies médiévales changerait fort, aussi, nos appréciations sur leur fondateurs. L'importance d'un Abélard est mince, comparée à celle du franciscain Alexandre de Halès, le créateur de la méthode employée par le plus grand penseur du Moyen-Age, S. Thomas; l'inventeur du Sic et Non n'est qu'un précurseur. De même, en face, non seulement de Jean Scot, mais aussi de Gerbert, de S. Anselme et de Jean de Salisbury, Alcuin et Heiric d'Auxerre, Bérenger de Tours et Abélard sont des hommes de second ordre. Raban Maur, l'élève d'Alcuin, Remi d'Auxerre, Roscelin et Guillaume de Champeaux sont inférieurs à tous les précédents, soit en valeur intrinsèque, soit quant à l'étendue de leur action, soit à ces deux points de vue.

La manière dont M. P. est conduit à diviser et à subdiviser l'histoire des philosophies médiévales, résulte immédiatement des vues que nọus venons d'exposer. La voici. - Du I. siècle av. J C., avec Philon, au VII. ap. J. C., se constituent tous les courants philosophiques dont sortiront les grandes doctrines qui s'épanouissent entre le VIII et la fin du XIII siècles. - Mais la première période (I siècle av., VIII siècle ap. J. C.) comprend trois parties. La première se termine au Concile de Nicée, en. 325; elle est marquée par une extrême vitalité des écoles platoniciennes, 
pythagoricjennes et chrétiennes, sans cesse en rapports les unes avec les autres. Elle aboutit à'la constitution définitive des dogmes fondamentaux du Christianisme. De 325 à 529, c'est à dire à la fermeture de l'Ecole d'Athènes par Justinien, l'activité philosophicothéologique est considérable encore, en Orient et en Occident. De 529 au VIII siècle, le Néoplatonisme achève de pénétrer théologies et philosophies. - Avec le VIII siècle finissant, que marque une première renaissance, commence la seconde période, qui se termine avec la première moitié du XVII siècle. - Cette seconde période peut aussi se subdiviser en trois parties. Pendant la première, qui va jusqu'au XIII siècle inclusivement, la spéculation juive, qui décline dès la mort de Maïmonide; la spéculation arabe, qui ne survit guère au XIII. siècle et dont l'Averroïsme est le moment le plus brillant; la Scolastique occidentale, enfin, inférieure en originalité ì la philosophie arabe, mais plus féconde qu'elle et qui progresse jusqu'à $\mathrm{S}$. Thomas, tandis que, dans l'orient chrétien, l'activité intellectuelle se fige ou s'éteint, offrent ì l'historien de la philosophie des sujets d'étude d'un puissant intérêt, mais aussid'une difficulté considérable; car alors, plus encore que dans la période précédente, toute école est telle qu'elle exige d'être étudiée dans ses rapports avec les autres et avec le mouvement scientifique ambiant. De la fin du XIII siècle à la prise de Constantinople en 1453 , la vie de l'esprit diminue partout, l'influence d'Aristote tend à restreindre celle du Néoplatonisme dans une certaine mesure; et si les Lniversités s'organisent en Occident, la décadence intellectuelle s'y poursuit néanmoins. Entre 1453 et la fin de la seconde période, une autre philosophie, aux formes très diverses, fille de l'humanisme et de la science renaissante, se fait jour dans les écoles catholiques, dans les écoles protestantes, et en dehors des milieux théologiques, car dès cette époque il existe de tels milieux. Mais l'esprit nouveau ne commence à vraiment prédominer qu'à partir de la révolution cartésienne. - Alors s'ouvre, parallèlement au mouvement intellectuel moderne, la troisième période de l'histoire des philosophies médiévales. M. P. étudie longuement les efforts croissants de la Scolastique, d'abord pour se maintenir en face de la philosophie indépendante et de la 
science expérimentale, puis pour repousser les conséquences théoriques et pratiques des courants d'idées issus de la Révolution française, enfin, et ceci n'est pas le moins curieux, pour s'assimiler toutes les conquêtes définitives de l'esprit humain et les faire entrer dans une synthèse puissante dont l'âme serait la vieille Scolastique elle-même. Tandis que celle-ci survivait, ou même survit encore, transformée, après une évolution continue, dans un grand nombre de métaphysiques actuelles, elle tâche de renaître, en l'intégrité de la forme qu'elle revêtit au XIII siècle, au sein de l'ècole néoscolastique qui repousse ces métaphysiquèes. M. P. suit les traces de la pensée du Moyen-Age jusque dans les débats politiques actuels; il voit, dans la tentative de Léon XIII pour restaurer la Scolastique, la preuve de l'accord intime - accord dont, il est vrai, doutent beaucoup de catholiques - de la religion romaine et de la spéculation médiévale.

Un des passages les plus importants du livre, est celui où M. P. dégage avec netteté la puissante, grandiose et poétique conception néoplatonico-chrétienne d'un univers où règne un dualisme radical. En haut, le monde divin, «régi par le principe de perfection»; en bas, le monde naturel «régi par les principes de contradiction et de causalité». Cette conception des choses étant acceptée, le problème capital, pour l'humanité, pourrait-il être autre que celui-ci: Comment peut s'opérer l'union de la nature et du divin? Pour en concevoir la possibilité, la Scolastique chrétienne se représente, au sein de la nature, une hiérarchie d'essences qui doit s'achever, au dessus d'elle, dans le monde supérieur. Pour réaliser cette union, on établit une hiérarchie ecclésiastique à laquelle il est logique de donner le pas sur les puissances temporelles; et l'éthique toute religieuse qui domine cette politique toute métaphysique, a pour base un tableau des valeurs calqué sur le tableau même des essences, disposées elles-même suivant leur rapport plus ou moins immédiat avec l'essence suprême. Cette conception explique, en dernière analyse, les idées politiques et morales fondamentales du Moyen-Age chrétien; elle est l'âme de toute sa philosophie, essentiellement théologique et néoplatonicienne sous le revêtement aristotélicien qui la dérobait à la vue des anciens historiens. 
De la longue étude à laquelle M. P. donne son Esquisse pour introduction, il espère à bon droit une confirmation nouvelle de l'idée qui s'impose de plus en plus aux savants en tout ordre de recherches, l'idée de la continuité dans l'évolution. Il n'y a pas plus d'hiatus dans l'histoire de la pensée que dans la succession des espèces; et il était vraiment étrange que notre érudition traitàt l'histoire de la philosophie comme si, entre l'Antiquité et les Temps modernes, tout l'immense intervalle fût chose à peu près négligeable. Comme toute autre époque, le Moyen-Age fut un moment nécessaire, et même relativement harmonieux, du développement de l'humanité. Il semble aussi à M. P. qu'en dépit de l'irrémédiable caducité d'une partie considérable de la pensée médiévale, quelque chose en demeurera, non pas ce qu'en veulent restaurer les Néoscolastiques, mais ce qui, de l'Idéal transcendant de jadis, peut être traduit en un «Idéal humain, de plus en plus élevé, de plus en plus accepté et poursuivi par tous». Cette dernière conclusion prêterait sans doute à la discussion, mais nous devons nous borner. M. P. nous y invite lui-même en nous montrant quelle tâche énorme l'histoire des idées et la psychologie de l'humanité doivent encore fournir, avant qu'il devienne possible de prophétiser avec quelque assurance le sort futur des écoles actuelles. Quoi qu'il en soit, son livre invite à penser que l'idéalisme contemporain, qui plonge par sa racine en une philosophie qui remonte à vingt sciècles et ne mourut jamais que pour renaitre, saura s'harmoniser dans l'avenir avec l'esprit scientifique, comme il sut s'harmoniser, dans le passé, avec la pensée naturaliste des Grecs. Revivre dans le présent le meilleur du passé est souverainement désirable, et le livre de $M$. Picavet nous donne cette joie de constater que la loi suprême de l'histoire des idées est conforme au væu suprême de l'esprit. 